University of Warwick institutional repository

This paper is made available online in accordance with

publisher policies. Please scroll down to view the document itself. Please refer to the repository record for this item and our policy information available from the repository home page for further information.

To see the final version of this paper please visit the publisher's website. Access to the published version may require a subscription.

Author(s: G. Gregori and D. O. Gericke

Article Title: A reduced coupled mode description for the electron-ion energy relaxation in dense matter

Year of publication: 2008

Link to published version: http://dx.doi.org/ 10.1209/0295-

5075/83/15002

Publisher statement: None 


\title{
A reduced coupled mode description for the electron-ion energy relaxation in dense matter
}

\author{
G. Gregori ${ }^{1,2}$ and D.O. Gericke ${ }^{3}$ \\ 1 Clarendon Laboratory, Department of Physics, University of Oxford, Parks Road, Oxford \\ OX1 3PU, United Kingdom \\ 2 Central Laser Facility, Rutherford Appleton Laboratory, Didcot OX11 OQX, United King- \\ dom \\ 3 Centre for Fusion, Space and Astrophysics, Department of Physics, University of War- \\ wick, Coventry CV4 7AL, United Kingdom
}

PACS 52.70.La -

PACS 71.10. $\mathrm{Ca}-$

PACS $61.10 . \mathrm{Eq}-$

\begin{abstract}
We present a simplified model for the electron-ion energy relaxation in dense twotemperature systems that includes the effects of coupled collective modes. It also extends the standard Spitzer result to both degenerate and strongly coupled systems. Starting from the general coupled mode description, we are able to solve analytically for the temperature relaxation time in warm dense matter and strongly coupled plasmas. This was achieve by decoupling the electron-ion dynamics and by representing the ion response in terms of the mode frequencies. The presented reduced model allows for a fast description of temperature equilibration within hydrodynamic simulations and an easy comparison for experimental investigations. For warm dense matter, both fluid and solid, the model gives a slower electron-ion equilibration than predicted by the classical Spitzer result.
\end{abstract}

Published: Europhysics Letters 83, 15002 (2008)

Introduction. - The energy equilibration in systems out of local thermodynamic equilibrium is of critical importance for the understanding of the quasi-equation of state, opacity, and optical response of astrophysical and laboratory plasmas. Transport properties such as heat conduction can be also very sensitive to the effectiveness of the energy transfer between electrons and ions. Nevertheless, the energy relaxation is often described by a simple Spitzer-like formula $[1,2]$ or its equivalent for systems with degenerate electrons derived by Brysk [3]. Such treatment neglects many important effects, in particular, collective modes. In denser systems, close collisions and the existence of a short range structure supported by the strong Coulomb forces must be also taken into account.

The effect of collective modes on the energy relaxation have been studied with different approaches. However, the magnitude of such effects is still under debate for solids (see, e.g., Refs. $[4,5])$ as well as for strongly coupled fluids [6-10]. Experimental verification of the relaxation times in dense matter is complicated by the fact that the relevant parameters are difficult to be measured directly. In fact, they are often just inferred from radiation-hydrodynamics simulations. Only a few experimental data points for the temperature relaxation times exist [11-13] and they indeed suggest much slower relaxation to 
equilibrium than predicted by the Landau-Spitzer model.

In the laboratory, dense two-temperature systems occur as a sub-picosecond transient state when solid targets are illuminated by intense short pulse lasers. During this process nonequilibrium conditions can be created by either laser ablation of the front surface thus launching a shock wave into the solid target, which preferentially heats the ion subsystem, or by transferring a larger fraction of the laser energy directly to the electrons, which typically happens in short-pulse laser-plasma interaction experiments [14].

Many dense laboratory plasmas are characterised by near-solid densities $\left(\rho \lesssim 1 \mathrm{~g} / \mathrm{cm}^{3}\right)$ and temperatures between a fraction to a few tenth of an electronvolt. This parameter region is often called warm dense matter (WDM) and exhibits states that have characteristics common to both solids and plasmas. This type of matter is also of particular interest for laboratory astrophysics, since it occurs in the core of large planets [15], and for studies in the context of inertial confinement fusion where it is created as a transient state in hohlraums [16].

WDM poses formidable challenges for the modelling of the energy relaxation processes since strongly coupled ion and degenerate electron physics has be to included self-consistently. The forces between the ions are usually expressed by the classical coupling parameter $\Gamma_{i i}=(Z e)^{2} / 4 \pi \epsilon_{0} a_{i} k_{B} T_{i}$, where $Z$ is the ion charge and $a_{i}=\left(3 / 4 \pi n_{i}\right)^{1 / 3}$ is the mean interion separation (or Wigner-Seitz radius) with $n_{i}$ being the ion density. Strongly coupled systems, where the Coulomb interactions dominate the average kinetic energy, i.e., $\Gamma_{i i} \gtrsim 1$, exhibit a long range structure (as in a solids) or well-pronounced short range correlations in the fluid regime [17]. In addition, the electron subsystem in WDM is in a state of partial degeneracy having an electron Fermi energy comparable to the thermal excitations, i.e., $T_{F} \sim T_{e}$, where $T_{F}=\hbar^{2}\left(3 \pi^{2} n_{e}\right)^{2 / 3} / 2 m k_{B}\left(n_{e}=Z n_{i}\right.$ is the electron density and $m$ the electron mass). Since both the Coulomb coupling parameter $\Gamma_{i i}$ and the degeneracy parameter $\Theta=T_{F} / T_{e}$ are in the order of unity or larger, the dynamics in nonequilibrium WDM cannot be described by the usual perturbative expansion techniques.

In this letter, we develop a reduced model that goes beyond the classical Spitzer formula taking into account strongly coupled ions, degenerate electrons and the coupled modes in the system. In a real plasma the energy relaxation is determined by both direct electron-ion interactions as well as indirectly by changes in the bound and free electronic configurations. In this work, we concentrate on the direct part; the description of the contributions from the excitation and ionisation has been discussed in Refs. [18-20] where only marginal ion heating related to these inelastic scattering processes was found. A complete description of a relaxing plasma would of course also include radiation transport and the hydrodynamical expansion.

Landau-Spitzer formula. - The relaxation towards equilibrium mainly occurs through electron-ion collisions and has been studied in several investigations $[6,8,10,21]$. The LandauSpitzer result for weakly coupled, classical plasmas is usually used as a reference. In this formulation, that assumes two ideal fluids, the relaxation is fully described by the relaxation time $[1,2]$

$$
\begin{aligned}
\tau_{e i}^{L S} & =\frac{3\left(4 \pi \epsilon_{0}\right)^{2} m M}{8 \sqrt{2 \pi} Z^{2} e^{4} n_{i} \Lambda}\left(\frac{m}{k_{B} T_{e}}+\frac{M}{k_{B} T_{i}}\right)^{-3 / 2} \\
& \sim \frac{3\left(4 \pi \epsilon_{0}\right)^{2} M\left(k_{B} T_{e}\right)^{3 / 2}}{8 \sqrt{2 \pi} Z^{2} e^{4} m^{1 / 2} n_{i} \Lambda}
\end{aligned}
$$

where $M$ is the ion mass ( $M \gg m$ has been used in the second step), and $\Lambda=\ln \left(b_{\max } / b_{\min }\right)$ is the Coulomb logarithm defined in terms of the minimum $\left(b_{\min }\right)$ and maximum $\left(b_{\max }\right)$ impact parameters. The ad hoc cutoff parameters $b_{\min }$ and $b_{\max }$ in the Coulomb logarithm are usually set to be the distance of closest approach $\rho_{\perp}=Z e^{2} / 4 \pi \epsilon_{0} m v_{e}^{2}$, with the thermal electron velocity $v_{e}=\left(k_{B} T_{e} / m\right)^{1 / 2}$, and the classical electron Debye length $\lambda_{D e}=\left(\epsilon_{0} k_{B} T_{e} / e^{2} n_{e}\right)^{1 / 2}$, respectively. 
The Landau-Spitzer approach sufficiently describes binary collisions in the low density limit, but it fails at higher densities as $\Lambda$ becomes negative. In order to prevent this behaviour, modified forms for the Coulomb logarithm have been suggested [21-23]. Good agreement between exact quantum binary collision approach and Eq. (1) could be obtained when the Coulomb logarithm is redefined as $\Lambda=0.5 \ln \left(1+b_{\max }^{2} / b_{\min }^{2}\right)$ and the lower cutoff $b_{\min }$ is set to be a quadratic interpolation of the de Broglie wave length $\lambda_{b}=\hbar / 2 m v_{e}$ and the distance of closest approach, i.e., $b_{\min }=\left(\lambda_{b}^{2}+\rho_{\perp}^{2}\right)^{1 / 2}$ [21]. Fitting the value of $\Lambda$ to the full T-matrix results yields even well-defined relaxation times beyond the usual limit of $\Lambda \gtrsim 3$. However, this method considers only binary collisions in a nondegenerate plasmas; it neither describes the partially to highly degenerate electrons in WDM nor does it include the structure in the ionic subsystem nor collective modes.

Reduced description of collective modes. - To extend the binary collision approach, one has to consider collective modes in the system. While the electron response might be in first order always described by the model of a free electron gas, the ions in WDM must be treated as a strongly coupled fluid or, for higher coupling, they may form a lattice. Of course these qualitatively different spacial arrangements give rise to a different mode structure that is acoustic or phonon like. Indeed, the electron-ion equilibration in solid WDM is best described by electron-phonon interactions [4].

The consideration of coupled collective modes in two-temperature electron-ion systems is predicted to yield a considerably less effective energy transfer $[4,6]$ than the Landau-Spitzer formula (1). However, the related formalism requires the a double integration over the very spiky density response functions of the system. The evaluation is therefore numerically too demanding to be included into a hydro-dynamic description of relaxing matter as well as for an easy comparison with experimental data. For that reason, we attempt here a reduced description that might also lead to a deeper understanding of the underlying physics.

We start our analysis by describing the electron-ion energy relaxation rate using the Balescu-Lenard formalism [24, 25]

$$
\begin{aligned}
\frac{d E_{e}}{d t}= & -4 \hbar \int \frac{d \mathbf{k}}{(2 \pi)^{3}} \int \frac{d \omega}{(2 \pi)} \omega\left|\frac{V_{e i}(k)}{\epsilon(k, \omega)}\right|^{2} \operatorname{Im} \chi_{e e}(k, \omega) \\
& \times \operatorname{Im} \chi_{i i}(k, \omega)\left[n_{B}\left(\frac{\hbar \omega}{k_{B} T_{e}}\right)-n_{B}\left(\frac{\hbar \omega}{k_{B} T_{i}}\right)\right],
\end{aligned}
$$

where $E_{e}$ is the thermal energy of the electron, $\epsilon(k, \omega)$ is the full dielectric function of the electron-ion system, which include both electron and ion screening, $\chi_{j j}(k, \omega)$ are the density response functions of the species $j$, and $n_{B}(x)=[\exp (x)-1]^{-1}$ denotes the Bose function that is the occupation number of the collective excitations in each subsystem. Electron and ion subsystems are assumed to be in equilibrium at their respective temperatures. The two subsystems interact through a dynamically screened, effective potential $V_{e i}(k) / \epsilon(k, \omega)$, where $V_{e i}(k)=Z e^{2} / \epsilon_{0} k^{2}$ is the pure Coulomb potential.

Expression (2) is the weak coupling limit of the coupled mode formula derived by Dharma-wardana \& Perrot [6]. In this limit, the dielectric function has the form

$$
\epsilon(k, \omega)=1-\sum_{j} V_{j j}(k) \chi_{j j}^{0}(k, \omega),
$$

where $V_{j j}(k)$ is the pure Coulomb potential between the particles of the same species $j$ and $\chi_{j j}^{0}$ is their free particle density response function.

For the further analysis, we assume that the form of the expression (2) holds also in the strongly coupled regime. However, the dielectric function $\epsilon$ is in this case modified by local field corrections that describe the strong coupling effects [17]. As it will become clear in the further analysis, we do not need the correct (unknown) form of the dynamic local field corrections neither for the ions nor for the electrons, but only the dispersion relation of 
the mode spectrum which is in contrast to the direct evaluation of the coupled mode form derived by Dharma-wardana \& Perrot [6].

As in the analysis of Hazak et al. [8], we use the fact that Eq. (2) can be substantially simplified since the time scales of the electron and ion dynamics are very different and, therefore, the spectra of the density response functions $\chi_{e e}$ and $\chi_{i i}$ barely overlap. In particular, it is reasonable to treat the electron susceptibility in the low frequency limit

$$
\operatorname{Im} \chi_{e e}(k, \omega)=\left.\omega \frac{\partial \operatorname{Im} \chi_{e e}(k, \omega)}{\partial \omega}\right|_{\omega=0} .
$$

Since the relevant frequency scale which is defined by the ion response is very low, the Bose factors can also be expanded, e.g., $n_{B} \sim k_{B} T_{j} / \hbar \omega$. This reflects the fact that thermalization occurs over many collisions which each contribute only a small energy transfer, i.e., $\hbar \omega \ll k_{B} T_{j}$ (with $j=e$ or $i$ ). With these well-fulfilled approximations, Eq. (2) reduces to

$$
\begin{aligned}
\frac{d E_{e}}{d t}= & -2 k_{B}\left(T_{e}-T_{i}\right) \int \frac{d \mathbf{k}}{(2 \pi)^{3}} V_{e i}^{2}(k) \\
& \times\left.\frac{\partial \operatorname{Im} \chi_{e e}(k, \omega)}{\partial \omega}\right|_{\omega=0} \int \frac{d \omega}{\pi} \omega \frac{\operatorname{Im} \chi_{i i}(k, \omega)}{|\epsilon(k, \omega)|^{2}} .
\end{aligned}
$$

As it becomes clear from the derivation of the Eq. (2), the response functions $\chi_{j j}(k, \omega)$ in the nominator describe the pure kinematics of the components [25]. Accordingly, they should be always used as free particle response functions, i.e., $\chi_{j j}(k, \omega)=\chi_{j j}^{0}(k, \omega)$. In the nondegenerate limit, we have

$$
\left.\frac{\partial \operatorname{Im} \chi_{e e}^{0}}{\partial \omega}\right|_{\omega=0}=-\left(\frac{\pi m}{2 k_{B} T_{e}}\right)^{1 / 2} \frac{n_{e}}{k_{B} T_{e}} \frac{1}{k} e^{-k^{2} \lambda_{b}^{2} / 8}
$$

and for a degenerate Fermi gas, one obtains

$$
\left.\frac{\partial \operatorname{Im} \chi_{e e}^{0}}{\partial \omega}\right|_{\omega=0}=-\frac{m^{2}}{4 \pi \hbar^{3} k} f_{e}(k)
$$

where $f_{e}$ is the Fermi distribution. Similarly, the free particle response of the ions is described by the semiclassical formula (6) by including the ion properties.

As shown by Hill et al. [26], experiments on solid and liquid metals indicate that the presence of the ionic background only slightly affects the electronic density response $\chi_{e e}$, and the assumption $\chi_{e e} \approx \chi_{e e}^{0}$ safely holds. In this case, $\chi_{e e}^{0}$ can be easily obtained within the jellium model [17].

In contrast, the dielectric response function $\epsilon(k, \omega)$ contains the information of the fully correlated medium. Since the ionic susceptibility effectively restricts the $\omega$-integral to very small frequencies, the dielectric function is mainly determined by the ion acoustic modes. These modes, defined by zeros in the real part of the total dielectric function, give rise to sharp peaks in the screened potential $V_{e i} / \epsilon$. Using an ansatz similar to the plasmon pole approximation, the inverse dielectric function can be written as [27-30]

$$
\frac{1}{\epsilon(k, \omega)}=\frac{k^{2}}{k^{2}+\left[1-G_{e e}(k)\right] k_{D e}^{2}}\left(\frac{\omega^{2}}{\omega^{2}-\omega_{k}^{2}}\right),
$$

where $k_{D e}$ is the inverse of the screening length [30] $\left(k_{D e}=1 / \lambda_{D e}\right.$ for a classical plasma), and $G_{e e}(k)$ is the local field correction for the electron subsystem. While in the relevant WDM regimes the ions are strongly coupled $\left(\Gamma_{i i} \gg 1\right)$, the electrons are only weakly coupled due to their lower charge state and the quantum exchange contributions in the kinetic energy (Fermi energy). Thus the correction introduced by $G_{e e}(k)$ is not expected to be significant. 
The frequencies $\omega_{k}$ denote the eigenfrequencies of the ion acoustic or phonon modes. These modes are strongly affected by correlations as is highlighted by the different mode structure at the two sites of the melting line. Their position and type also depends on the mutual interaction with the electrons (coupled modes). An important point implicit in Eq. (8) is that for small energy transfers between electrons and ions (i.e., $\omega<\omega_{k}$ ) the effective interaction potential $V_{e i} / \epsilon$ can be strongly suppressed by virtue of the additional dynamical screening from the ions.

Substituting the ansatz (8) for the dielectric function into the expression (5), one can perform the $\omega$-integration analytically. We then obtain

$$
\begin{aligned}
\frac{d E_{e}}{d t}= & -\frac{Z^{2} e^{4} n_{i} k_{B}\left(T_{e}-T_{i}\right)}{\epsilon_{0}^{2} \pi^{2} M} \\
& \times\left.\int d k \frac{k^{4} Y\left(c_{s} / v_{t i}\right)}{\left[k^{2}+\left[1-G_{e e}(k)\right] k_{D e}^{2}\right]^{2}} \frac{\partial \operatorname{Im} \chi_{e e}^{0}}{\partial \omega}\right|_{\omega=0},
\end{aligned}
$$

where $v_{t i}=\sqrt{k_{B} T_{i} / M}$ is the thermal speed of the ions, $c_{s}=\omega_{k} / k$ is the sound speed of the ionic modes. For $Y=1$, the integral in $k$-space represents a generalised form of the Coulomb logarithm which has the advantage that no (hard) bounds in the integration need to be introduced to assure convergence. The integral is however effectively cut off at similar points namely the thermal electron (de Broglie) wave length for large $k$ values (or small distances) and the electron screening length at small $k$ (or large distances). A similar form was first derived by Hazak et al. [8] using the Fermis golden rule approach that neglects the coupled mode contributions (therefore $Y=1$ in their formulation). form

The coupled mode effects are condensed in the correction factor $Y\left(c_{s} / v_{t i}\right)$ which has the

$$
\begin{aligned}
Y(x)= & \mid 1+2 x^{2}-\frac{x^{4}}{2}+\frac{\sqrt{2 \pi}}{4} e^{-x^{2} / 2} x^{3}\left(x^{2}-5\right) \\
& \times\left(\frac{2}{\sqrt{\pi}} \int_{0}^{x / \sqrt{2}} e^{u^{2}} d u-i\right) \mid
\end{aligned}
$$

for $k<k_{c}$ and $Y(x)=1$ otherwise. Here, $k_{c}$ denotes the critical wave number above which the modes cease to exit. The function $Y\left(c_{s} / v_{t i}\right)$ is plotted in Fig. 1. We notice that a considerable reduction of the relaxation rate can be expected when both $c_{s} / v_{t i}$ and $k_{c}$ are large. We should also point out that the function $Y(x)$ can be written in terms of tabulated integrals [31], and it can be thus easily embedded in a full radiation-hydrodynamics calculation of heat transport in a laser-plasma simulation.

Eq. (9) can be further simplified in the case of semi-classical systems with hot, nondegenerate electrons and neglecting the small correlation effects in the electron energy, i.e., $E_{e}=3 n_{e} k_{B} T_{e} / 2$. Exploiting the fact that the $k$-integral is naturally split into two different regions separated by the critical wave number $k_{c}$, we can formulate the temperature relaxation process in a Spitzer-like expression

$$
\frac{d T_{e}}{d t}=\frac{T_{e}-T_{i}}{\tau_{e i}},
$$

with

$$
\begin{aligned}
\frac{1}{\tau_{e i}}= & \frac{8 \sqrt{2 \pi} Z^{2} e^{4} n_{i} \Lambda(\infty)}{3\left(4 \pi \epsilon_{0}\right)^{2} m M}\left(\frac{m}{k_{B} T_{e}}\right)^{3 / 2} \\
& \times\left[1+\frac{\Lambda\left(k_{c}\right)}{\Lambda(\infty)}\left[Y\left(c_{s} / v_{t i}\right)-1\right]\right], \\
= & \frac{1}{\tau_{e i}^{*}}\left[1+\frac{\Lambda\left(k_{c}\right)}{\Lambda(\infty)}\left[Y\left(c_{s} / v_{t i}\right)-1\right]\right],
\end{aligned}
$$


where we defined

$$
\Lambda(x)=\int_{0}^{x} \frac{d k}{k} \frac{k^{4} e^{-k^{2} \lambda_{b}^{2} / 8}}{\left[k^{2}+\left[1-G_{e e}(k)\right] k_{D e}^{2}\right]^{2}} .
$$

We notice that $\Lambda(\infty)$ corresponds to the generalised form of the Coulomb logarithm. The factor

$$
W=1+\frac{\Lambda\left(k_{c}\right)}{\Lambda(\infty)}\left[Y\left(c_{s} / v_{t i}\right)-1\right]
$$

includes the coupled mode correction to the Landau-Spitzer relaxation time, i.e., $\tau_{e i}=$ $\tau_{e i}^{*} / W$. Clearly, when $W=1$ a generalised form of Eq. (1) is obtained, with the only difference that in this case the generalised Coulomb logarithm $\Lambda$ allows for the static correlations to be accounted for. The full coupled mode dynamics is therefore enclosed solely in the function $W$.

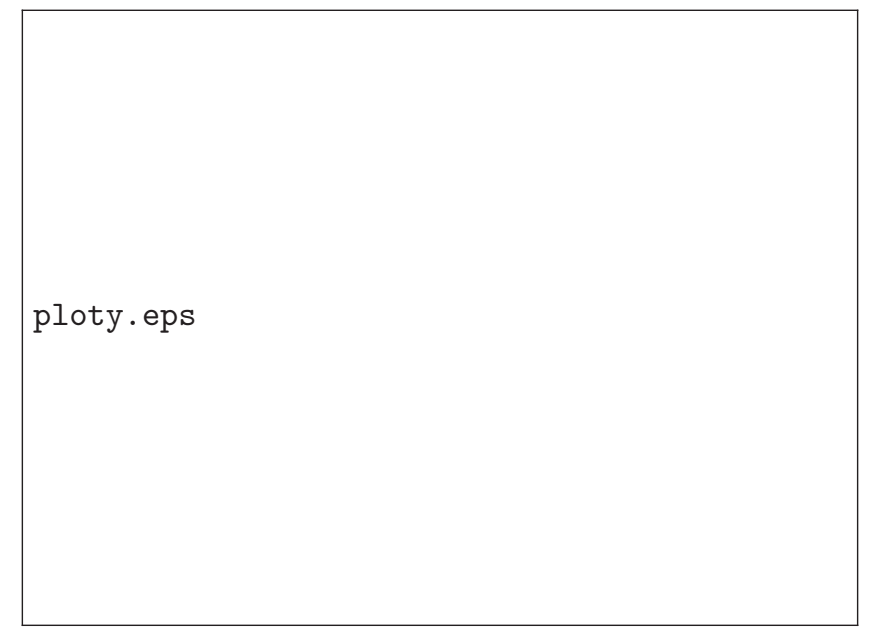

Fig. 1: The function $Y\left(c_{s} / v_{t i}\right)$ describing the coupled mode corrections (see text). The plasmonpole approximation, which is used to derive the approximate form of the relaxation time, is only valid for $c_{s} / v_{t i}>1$.

Eq. (14) contains two parameters: the sound speed $c_{s}$ and the sound attenuation length $1 / k_{c}$. The detailed form of these will depend on the particular conditions under investigation.

Dispersion relation for the ion modes. - The magnitude of the coupled mode effects in our reduced description of the energy transfer rate Eqs. (9) and (12) is fully determined by the position of the ion modes $\omega_{k}$ and the range in $k$ space, where these modes exist. In the following, we want to discuss three different systems with very different ionic mode structures: i) a weakly coupled ion subsystem (gas-like plasma); ii) WDM with ions that form a strongly coupled fluid (dense plasmas); and finally iii) warm dense matter states with ions frozen into a lattice (heated solids). In all of these cases, the mode structure can be characterised by the sound speed $c_{s}$, i.e., $\omega_{k}=c_{s} k$ for $k<k_{c}$.

Let us start with the well-known case of a gas-like plasma. Here, the relevant ion modes are the ion acoustic excitations with the well-known sound speed

$$
c_{s}=\sqrt{Z k_{B} T_{e} / M} .
$$

In order to have $c_{s}>v_{t i}$, as required by the form for the dielectric function (8), $Z T_{e} / T_{i} \gg 1$ must hold which typically applies for most systems heated by lasers. In this weakly coupled, gas-like state, both electrons and ions can be described by the random phase approximation. 
Accordingly, the zeros in the dielectric function occur for $k<k_{c} \approx k_{D i}$, where $k_{D i}=$ $\left(Z^{2} e^{2} n_{i} / \epsilon_{0} k_{B} T_{i}\right)^{1 / 2}$ is the inverse of the ion Debye screening length.

If the ions form a strongly coupled fluid system, that is for $\Gamma_{i i}>1$, the sound speed is modified by the correlations as expressed in the static local field corrections $G_{i i}(k)$

$$
c_{s}=\sqrt{\left(Z k_{B} T_{e} / M\right)\left[1-G_{i i}(k)\right]} .
$$

The static local field corrections are connected to the ion-ion structure factor $S_{i i}(k)$. Assuming an ion subsystem that is linearly screened by the electrons (Yukawa model), one obtains [17]

$$
G_{i i}(k)=\frac{1}{\epsilon_{e}(k, 0)}-\frac{k}{k_{D e}}\left[\frac{1}{S_{i i}(k)-1}\right],
$$

where $\epsilon_{e}$ is the electronic dielectric function in RPA. The ionic structure factor $S_{i i}(k)$ can be easily calculated from classical numerical simulations (molecular dynamics or Monte Carlo) or hypernetted chain calculations. The latter can also be used to go beyond the linear screening model. Note that the sound speed becomes here $k$ dependent, and the calculation of the correction factor requires the full numerical solution of the integral in k-space. On the other hand, $G_{i i}(k)$ scales as $k^{2}$ as $k \rightarrow 0$ [17], and for values of the sound attenuation that are sufficiently small, the approximation $G_{i i}(k) \sim 0$ is often sufficient.

For strongly coupled plasmas in the hydrodynamic limit, the inverse sound attenuation has been shown to hold [32]

$$
k_{c}=2 c_{s}\left(\frac{\gamma-1}{\gamma} \frac{\kappa}{n_{i} C_{v}}+\frac{4}{3} \frac{\eta}{n_{i} M}\right)^{-1},
$$

where $\kappa$ is the thermal conductivity of the ionic system, $\eta$ the viscosity and $\gamma=C_{p} / C_{v}$ the ratio of the specific heats. $C_{p}$ is the specific heat at constant pressure and $C_{v}$ the specific heat at constant volume. The transport properties of the ionic fluid can be estimated from fits for one component plasmas, i.e., by assuming the ions are immersed in a rigid neutralising background of negative charges $[33,34]$

$$
\begin{aligned}
\frac{\kappa}{k_{B} n_{i} \omega_{p i} a_{i}^{2}}= & \frac{2.02037}{\Gamma_{i i}}+\frac{\Gamma_{i i}}{195.006}+0.1655 \\
\frac{\eta}{M n_{i} \omega_{p i} a_{i}^{2}}= & \frac{1.06154}{\Gamma_{i i}^{29 / 18}}\left[\frac{\left(0.49-\frac{2.23}{\Gamma_{i i}^{1 / 3}}\right) \Gamma_{i i}^{3 / 2}}{5 \sqrt{3} \pi}+1\right]^{2} \\
& +\frac{\sqrt{\Gamma_{i i}}}{15 \sqrt{3 \pi}}
\end{aligned}
$$

where $\omega_{p i}=\left(Z^{2} e^{2} n_{i} / \epsilon_{0} M\right)^{1 / 2}$ is the ion plasma frequency. The excess (non-ideal) contributions to the specific heats are $[35,36]$

$$
\begin{aligned}
\frac{C_{v}^{e x}}{k_{B}} & =-\Gamma_{i i}^{2} \frac{\partial}{\partial \Gamma_{i i}} \frac{u_{e x} / N k_{B} T_{i}}{\Gamma_{i i}} \\
\frac{C_{p}^{e x}}{k_{B}} & =\frac{C_{v}^{e x}}{k_{B}}+\frac{1}{3} \frac{\left(C_{v}^{e x} / k_{B}\right)^{2}}{4\left(u_{e x} / N k_{B} T_{i}\right)-\left(C_{v}^{e x} / k_{B}\right) / 3},
\end{aligned}
$$

where $u_{e x}$ is the excess energy due to correlations. It can be obtained from parametric fits of Monte Carlo calculations [37]. The total contribution of the specific heats is then $C_{v}=3 / 2+C_{v}^{e x}$ and $C_{p}=5 / 2+C_{p}^{e x}$. The formulas for the transport coefficient and the excess energy can also be generalised for a screened Yukawa liquid [34,38]. 
The small frequency phonon waves in solids are determined by the Debye temperature $T_{D}$ of the lattice. The corresponding sound speed of these phonon modes is [39]

$$
c_{s}=\frac{k_{B} T_{D}}{\hbar}\left(6 \pi^{2} n_{i}\right)^{-1 / 3} .
$$

The energy scale is set here by the Debye temperature $T_{D}$ which represents the temperature above which all the phonons become thermally activated [29] and the electron-phonon interaction must be treated in detail [40]. It depends on the crystal structure and, similarly to a strongly coupled plasma, on the details on the ion-ion correlations. In the case of a solid, the thermal conductivity, viscosity and heat capacities can be directly obtained from tabulated data and, again, Eq. (18) can be used to obtain the inverse sound attenuation length.

Discussion and concluding remarks. - The reduced coupled mode formulae (9) and (12) are the main result of this work. They show that a simple Landau-Spitzer form of the energy relaxation can still be retained in the WDM region while the coupling of electron and ion modes is described in terms of an additional correction factor which is a functional of the degree of ionic correlations in the system. The simplified coupled mode formulae generalise the original Spitzer model in three respects: they can be applied to systems with i) strongly coupled ions and ii) degenerate electrons without an unphysical behaviour (negative relaxation times due to hard cutoffs in the Coulomb logarithm) and iii) they include the effect of coupled collective modes. It is interesting to notice that the improved Coulomb logarithm, which is similar as in the Fermi's golden rule description of Refs. [8,25], yields the largest effect and opposes the coupled mode effect. In WDM, the related correction can be orders of magnitude since it improves the approach from being inapplicable to the right order of magnitude. The coupled collective modes give rise to another correction in the order of two to ten (see Fig. 1). Thus our reduced approach yields similar coupled mode corrections as the full coupled mode description of Ref. [6] (compare, e.g., FRG and CM in the figure of Ref. [42]).

There are regimes where the energy relaxation between electrons and ions can be considerably suppressed. Let us consider as an example the case of a dense beryllium plasma, as investigated in the recent experiment by Glenzer et al. [41]. Using the experimentally measured electron density and ionisation state $\left(n_{e} \sim 3.3 \times 10^{23} \mathrm{~cm}^{-3}, Z=2.7\right)$, we obtain $\tau_{e i} \sim 1.7 \times \tau_{e i}^{*}$ from the present model when $T_{e} / T_{i} \sim 2$; this factor of 1.7 is the pure coupled mode effect. One should however keep in mind that, due to strong coupling and degeneracy present in this case, $\tau_{e i}^{*}$ is about a factor of twenty smaller than the usual Spitzer relaxation time. Based on this analysis, we speculate that this system is in a state where inhibition of the energy relaxation could be expected and it would pose as a perfect candidate for future investigations of energy transfer processes in WDM.

The results presented in this work are very important for the understanding of heat transport and conduction mechanisms in dense systems. In addition, the formula derived is relatively simple and it can be easily implemented into radiation-hydrodynamics simulation codes designed for WDM where it should significantly improve the predictive capabilities of equation of state properties. This is clearly an important step for the success on inertial confinement fusion (ICF) research [16] and the understanding of laboratory astrophysics experiments [15].

$$
* * *
$$

We thankfully acknowledge stimulating discussions with Drs. O.L. Landen and S.H. Glenzer (Lawrence Livermore National Laboratory), Profs. R. Evans and S. Rose (Imperial College), and Dr. J. Daligault (Los Alamos National Laboratory). A special thank to 
Profs. R. Redmer and W.-D. Kraeft for their hospitality at the University of Rostock, Germany. This work was supported by funds of the Engineering and Physical Sciences Research Council and by the Science and Technology Facilities Council of the United Kingdom.

\section{REFERENCES}

1] Landau L. D., Phys. Z. Sowjetunion, 10 (1936) 154.

[2] Spitzer L., Physics of Fully Ionized Gases (Interscience, New York) 1967.

[3] Brysk H., Plasma Phys. , 16 (1974) 927.

[4] Dharma-Wardana M. W. C., Phys. Rev. Lett. , 66 (1991) 197.

[5] Sarma S. D. and Korenman V., Phys. Rev. Lett. , 67 (1991) 2916; Dharma-Wardana M. W. C., Phys. Rev. Lett. , 67 (1991) 2917; Senna J. R. and Sarma S. D., Phys. Rev. Lett. , 70 (1993) 2593; Xing D. Y. and Ting C. S., Phys. Rev. Lett. , 72 (1994) 2812; DharmaWardana M. W. C., Phys. Rev. Lett. , 72 (1994) 2811; Senna J. R. and Sarma S. D., Phys. Rev. Lett., 72 (1994) 2813.

6] Dharma-Wardana M. W. C. and Perrot F., Phys. Rev. E, 58 (1998) 3705.

[7] Perrot F. and Dharma-Wardana M. W. C., Phys. Rev. B, 62 (2000) 16536.

[8] Hazak et al. G., Phys. Rev. E , 64 (2001) 066411.

[9] Hazak G. and Argaman N., Phys. Rev. E , 69 (2004) 066407.

[10] Daligault J. and Mozyrsky D., Phys. Rev. E , 75 (2007) 026402.

[11] Celliers P., NG A. and Forsman A., Phys. Rev. Lett., 68 (1992) 2305.

[12] Ng A., Celliers P., Xu G. and Forsman A., Phys. Rev. E, 52 (1995) 4299.

[13] Riley D., Woolsey N. C., McSherry D., Weaver I., Duaoui A. and Nardi E., Phys. Rev. Lett. , 84 (2000) 1704.

[14] Atzeni S. and ter Vehn J. M., The Physics of Inertial Fusion (Oxford University Press, Oxford) 2003.

[15] Remington B. A., Drake P. R. and Ryutov D. D., Rev. Mod. Phys., 78 (2006) 755.

[16] Lindl J. D., Inertial Confinement Fusion (Springer-Verlag, New York) 1998.

[17] Ichimaru S., Rev. Mod. Phys., 54 (1982) 1017.

[18] Ohde T., Bonitz M., Bornath Th., Kremp D. and Schlanges M.,, Phys. Plasmas, 3 (1996) 1241.

[19] Bornath Th., Schlanges M. and Prenzel R.,, Phys. Plasmas, 5 (1998) 1485.

[20] Gericke D. O., Grubert G. K., Bornath Th. and Schlanges M.,, J. Phys. A, 39 (2006) 4727.

[21] Gericke D. O., Murillo M. S. and Schlanges M., Phys. Rev. E, 65 (2004) 036418.

[22] Lee Y. T. and More R. M., Phys. Fluids , 27 (1984) 1273.

[23] Lee C.-K. and Petrasso R. D., Phys. Rev. Lett., 70 (1993) 3059.

[24] Brown L. S., Preston D. L. and Singleton R. L., Phys. Rep., 410 (2005) 237.

[25] Gericke D. O., J. Phys. (Conf. Sect.), 11 (2005) 111.

[26] Hill et al. J. P., Phys. Rev. Lett. , 77 (1996) 3665.

[27] Frohlich H., Proc. Roy. Soc. A, 215 (1952) 291.

[28] Bardeen J. and Pines D., Phys. Rev. , 99 (1955) 1140.

[29] Ashcroft N. W. and Mermin N. D., Solid State Physics (Brooks Cole, Monterey, CA) 1976.

[30] Kremp D. and Schlanges M. and Kraeft W.-D., Quantum Statistics of Nonideal Plasmas (Springer-Verlag, Berlin) 2004.

[31] Press W. H., Teukolsky S. A., Vetterling W. T. and Flannery B. P., Numerical Recipes (Cambridge University Press, Cambridge, MA) 1994.

[32] Hansen J.-P. and McDonald I. R., Theory of Simple Liquids (Academic, London) 2000.

[33] Wallenborn J. and Baus M., Phys. Lett., 61A (1977) 35.

[34] Faussurier G. and Murillo M. S., Phys. Rev. E, 67 (2003) 046404.

[35] Hansen J.-P., Phys. Rev. A , 8 (1973) 338.

[36] Baus M. and Hansen J.-P., Phys. Rep. , 59 (1980) 1.

[37] Ichimaru S., Statistical Plasma Physics (Addison-Wesley, Reading, MA) 1991.

[38] Hamaguchi S., Farouki R. and Dubin D., Phys. Rev. E, 56 (1997) 4671.

[39] Bohm D. and Staver T., Phys. Rev. , 84 (1951) 836.

[40] Allen P. B., Phys. Rev. Lett. , 59 (1987) 1460. 
[41] Glenzer S. H., Gregori G., Lee R. W., Rogers F. J., Pollaine S. W. and Landen O. L., Phys. Rev. Lett. , 90 (2003) 175002.

[42] Dharma-wardana M. W. C., Phys. Rev. E, 64 (2001) 035401. 